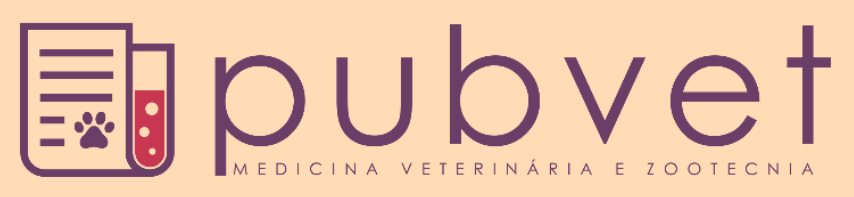

https://doi.org/10.31533/pubvet.v14n10a674.1-5

\title{
Avaliação do uso de anticoncepcionais em cães e gatos
}

\author{
Francisco Lima Silva ${ }^{1 *}$, Catarina Rafaela Alves da $\operatorname{Silva}^{20}$, Mariana Pacheco de $\operatorname{Sousa}^{30}$ (D), \\ Luciana Rangélia Malvina Souza de Castro $^{30}$, Artur Oliveira Rocha $^{3}{ }^{\circ}$, Tairine Melo Costa ${ }^{4}{ }^{\circ}$, \\ Thanisya Kelly de Paiva Brito ${ }^{\circ}{ }^{\circ}$, Eglesia Rodrigues Leite Fernandes ${ }^{4}{ }^{\circ}$, Kelline Emanuelle da \\ Rocha Rodrigues4 ${ }^{\circ}$
}

${ }^{I}$ Professor da Universidade Federal do Piauí, Departamento de Clínica e Cirurgia Veterinária. Teresina - PI. Brasil.

${ }^{2}$ Médica veterinária, Anestesiologista Hospital Veterinário Universitário, Universidade Federal do Piauí. Teresina - PI. Brasil.

${ }^{3}$ Graduandos em Medicina Veterinária, Universidade Federal do Piauí. Teresina - PI. Brasil.

${ }^{4}$ Médica veterinária autônoma Teresina - PI. Brasil.

*Autor para correspondência: E-mail: flimavet@hotmail.com

Resumo. O cão e o gato são espécies multíparas, com período gestacional curto e com alta prolificidade, logo muitos proprietários têm recorrido aos fármacos contraceptivos para a inibição e retardo do cio, pois são métodos barato e de fácil acesso. No entanto, muitos proprietários não são orientados sobre os efeitos adversos do uso do medicamento, e que tem provocado diversas patologias em animais de estimação, incluindo neoplasias, piometra, hiperplasias, aborto entre outros malefícios. Os progestágenos, megestrol, medroxiprogesterona e a proligestona, fazem parte dos recursos farmacológicos mais utilizados para o controle reprodutivo de cadelas. Este estudo objetivou avaliar na cidade de Pedro II, a casuística de utilização desses fármacos, a frequência de administração, os possíveis efeitos adversos e o conhecimento sobre os riscos que representa para o animal. Assim como foi identificado em outros estudos, em Pedro II também é comum o uso do contraceptivo, principalmente em gatas. O que pode se concluir com o trabalho é que a maioria dos proprietários não tem informação quanto aos efeitos negativos do uso de contraceptivos e sobre os benefícios da cirurgia de castração. Além da carência de médicos veterinários atuando na cidade.

Palavras chave: castração, fármacos contraceptivos, hiperplasia, neoplasia, pimetra

\section{Evaluation of contraceptive use in dogs and cats}

Abstract. Coxofemoral dysplasia (DCF) is an orthopedic condition characterized by the abnormal development of the hip joint and consequent degeneration of the articular components. The clinical signs when discrete are little evidenced by the tutors and their manifestation varies according to the age of the animal. The diagnosis is made through anamnesis, physical and radiographic findings. The treatment is performed with the intention of relieving pain and preventing the progression of degenerative joint disease. The present study aims to report the technique of acetabular denervation and pectineotomy as treatment of hip dysplasia in canine, where it can be observed a significant clinical evolution in the postoperative period of the animal.

Keywords: castration, contraceptive drugs, hyperplasia, neoplasia, pyometra

\section{Evaluación del uso de anticonceptivos en perros y gatos}

Resumen. El perro y el gato son especies multíparas, con un período gestacional corto y una alta proliferación, por lo que muchos propietarios han recurrido a medicamentos anticonceptivos para inhibir y retrasar el estro, ya que son métodos económicos y de fácil 
acceso. Sin embargo, a muchos propietarios no se les aconseja sobre los efectos adversos del uso del medicamento, y ha causado diversas patologías en las mascotas, que incluyen neoplasias, piometra, hiperplasia, aborto, entre otros daños. Las progestinas, megestrol, medroxiprogesterona y proligestone son parte de los recursos farmacológicos más utilizados para el control reproductivo de las perras. Este estudio tuvo como objetivo evaluar en la ciudad de Pedro II, la casuística del uso de estos medicamentos, la frecuencia de administración, los posibles efectos adversos y el conocimiento sobre los riesgos para el animal. Como se identificó en otros estudios, el uso de anticonceptivos en la ciudad de Pedro II también es común, especialmente en gatos. Lo que se puede concluir del trabajo es que la mayoría de los propietarios no tienen información sobre los efectos negativos del uso de anticonceptivos y los beneficios de la cirugía de castración. Además de la falta de veterinarios que trabajan en la ciudad.

Palabras clave: castración, anticonceptivos, hiperplasia, neoplasia, piometra

\section{Introdução}

Cadelas e gatas são animais pluríparas, de curto período gestacional, aproximadamente 60 dias, com alto número de filhotes por gestação e que iniciam a puberdade com idade média de 6 meses, no entanto gatas entram em maturidade sexual com 4 meses. Nas gatas, ao contrário de outras espécies, a manipulação do ciclo estral não é fácil. A maioria das gatas domésticas atinge a puberdade entre 8 a 13 meses de idade, mas existe uma variação considerável (Cáceres, 2004). Esta espécie apresenta ovulação induzida pelo coito, apresenta ciclo estral associado ao fotoperíodo positivo (Feldman et al., 2014).

O controle reprodutivo mais recomendado é a esterilização, obtida por meio de cirurgias de castração, sendo mais eficaz e seguro para impedir a reprodução; porém, os medicamentos contraceptivos (também conhecidos como anticoncepcionais) estão sendo utilizados com frequência com essa finalidade, que além de baixo custo são facilmente encontrados em casas de rações e lojas do gênero (Bocardo et al., 2008; Dias et al., 2013; Fernandes et al., 2020; Filgueira et al., 2008; Fonseca et al., 2014; Rossi et al., 2013; Viana et al., 2012).

O uso indiscriminado pode ocasionar sérios distúrbios reprodutivos, como o surgimento de piometra, hiperplasia endometrial cística, hiperplasia das glândulas mamárias, neoplasias mamárias e pode ocasionar a morte do feto se usado no período gestação (Gabaldi, 1998; Papich, 2012).

Em detrimento do uso comum, inadvertido e perigoso dos fármacos contraceptivos em pequenos animais, o presente estudo objetivou avaliar por meio de questionário feito aos proprietários de cães e gatos da zona urbana da cidade de Pedro II- PI, a casuística de utilização desses fármacos, a frequência de administração e os possíveis efeitos adversos apresentados. Além de orientar e conscientizar a população dos riscos de tais fármacos, incentivando a realização da esterilização cirúrgica como melhor método contraceptivo permanente.

\section{Relato do caso clínico}

A pesquisa foi realizada em quatro bairros, da zona urbana, no município de Pedro II no estado do Piauí, localizado a nordeste do estado do Piauí, com área de $1.948 \mathrm{~km} 2$, estando sua sede a $04^{\circ} 25^{\prime} 29^{\prime \prime}$ L.S. e $41^{\circ} 27^{\prime} 31^{\prime \prime}$ W.Gr. Com a população de 37.496 habitantes segundo o último senso do IBGE (2010).

A população alvo da pesquisa foram os proprietários de cães e gatos em bairros diversificados da zona urbana do município de Pedro II- PI, totalizando 70 pessoas entrevistadas.

Trata-se de um estudo descritivo do tipo transversal, quantitativo com aplicação de questionário em 8 dias consecutivos, elaborado para investigar sobre o uso de fármacos contraceptivos em cães e gatos e o conhecimento dos proprietários da sobre os mesmos na cidade de Pedro II- PI.

Foi elaborado um questionário, com inquirições para 70 proprietários de cães e gatos, no período de 7 a 14 de junho do ano de 2015. O questionamento baseou-se nas seguintes perguntas: $\mathrm{O}$ animal que possui, se cão ou gato, sexo, quantidade de animais, castrados ou não, administração ou não de fármacos contraceptivos, sendo que sobre os medicamentos interrogou-se, para quem já o utilizou, o nome (comercial e princípio ativo), frequência, onde adquiriu e quem aplicou o fármaco, presença ou não de 
efeitos colaterais ou outras afecções após tais aplicações, conhecimento sobre os riscos ao uso do fármaco, e por último se o proprietário castraria ou não seu animal, se não, quais os motivos.

Após a realização do questionário, foi orientado aos proprietários a castrarem seus animais e não utilizarem fármacos contraceptivos, devido os riscos que essas medicações podem causar na saúde dos mesmos.

\section{Resultados e discussão}

Após a análise do questionário com 70 proprietários de cães e gatos na cidade de Pedro II- PI, resultou em um total de 120 animais domiciliados, sendo 58 cães (33 machos e 25 fêmeas) e 62 gatos ( 38 machos e 24 fêmeas), de várias raças e idades. Em relação à espécie, o número de felinos nas residências, 62 $(51,7 \%)$ sobrepõe sobre a de caninos, $58(48,3 \%)$. Ao verificar o sexo que mais prevalece, há um número maior de pessoas que possuem cães e gatos machos, $71(59,1 \%)$ nas residências em relação àquelas que possuem fêmeas, $49(40,9 \%)$.

Verificou-se que $(35,7 \%)$ dos proprietários (Gráfico 1) usam ou usaram fármacos contraceptivos como meio de prevenir o acasalamento e subconsequente gestação indesejada. Concordado com o que disse Oliveira \& Marques-Júnior (2006) onde existe grande aceitação da comunidade para o uso destes medicamentos principalmente por serem de baixo custo e encontrados com facilidade.

\section{Uso ou não de anticoncepcionais}

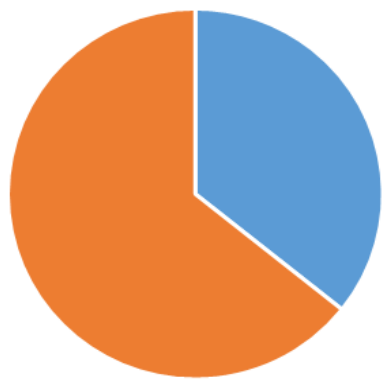

- SIM - NÃO

Gráfico 1. Relato do uso ou não de anticoncepcionais, através de questionários, aplicados à proprietários de animais de companhia na cidade de Pedro II- PI.

Dos animais dos proprietários, $118(98,4 \%)$ não eram castrados e apenas uma cadela e uma gata submeteram à cirurgia de $\mathrm{OSH}$, do tipo patológica e a outra eletiva, respectivamente. As cirurgias foram realizadas por médicos veterinários na cidade Teresina

Os proprietários das felinas são os que mais se utilizam do fármaco $(25,7 \%)$, seguido dos de cadela $(10 \%)$. A ligação do uso frequente em gatas, se justifica pelo fato da gata doméstica ser classificada como poliéstrica estacional fotoperíodica positiva. Em condições naturais, poliéstrica sazonal em regiões abaixo e acima dos trópicos e segundo Johnston et al. (2001 e Kustritz (2011), ela poliéstrica continua em regiões próximas à Linha do Equador. Romagnoli (2003) descreve que a maioria das gatas, atingem a puberdade quando o seu peso atinge igual à dois terços ou superior ao de uma gata adulta, $\mathrm{e}$ isso pode ocorrer entre 5 a 10 meses de idade, ou ser precoce ou tardiamente a esse período, ou seja, até os 21 meses (Romagnoli, 2003). Já Oliveira \& Marques Júnior (2006) ao controle da fêmea, e pouco se tem estudado quanto à esterilização do macho. Entretanto, cada macho intacto é um reprodutor em potencial. De forma que a esterilização de um grande número de machos contribui para o decréscimo do número de fêmeas gestantes.

Sobre o uso medicamento, nenhum proprietário soube informar o nome comercial (100\%). Em relação à frequência de uso, observou-se que foi aplicado uma vez na maioria dos animais (61\%), enquanto (39\%) aplicaram duas vezes ou mais. Todos afirmaram que produto foi adquirido em lojas de ração, sem presença do médico veterinário, e que a aplicação do medicamento foi feita, na maioria dos animais, pelo funcionário da casa de ração $(75 \%)$, outras realizadas em casa $(25 \%)$, e nenhuma aplicação 
por veterinário, esses dados vão de acordo com Neves et al.,(2003), quando ele diz que, os progestágenos e estrógenos são os contraceptivos mais utilizados e aplicados por técnicos não qualificados, que não respeitam a dose, o período de anestro, gestação e o peso do animal, favorecendo assim os inúmeros efeitos adversos. Os efeitos colaterais observados aos animais que receberam a aplicação, apenas 1\%, ou seja, 1 proprietário observou alteração, no caso uma piometra aberta em cadela, meses após a aplicação.

A maioria dos entrevistados, $(55,7 \%)$, não castraria seu animal, principalmente por motivos financeiros $(32,7 \%)$, seguido de medo do procedimento provocar danos e sofrimento ao animal $(20,0 \%)$ e outros motivos (3\%). Enquanto, uma menor parcela dos entrevistados castraria o seu animal $(44,3 \%)$ se houvesse, principalmente, clínicas de procedimento na cidade ou campanhas de castração (Gráfico 2). Esses resultados se assemelham ao estudo realizado por Silva et al. (2009), no bairro Buenos Aires em Teresina-PI, onde relata o tabu que muitos têm em relação ao controle reprodutivo dos seus animais, pois foram 77 entrevistados, e 65\% não castraria seu animal, por alegar poucos recursos financeiros, por medo de que seus animais sintam dor, ou que os mesmo fiquem acima do peso ou "preguiçosos" perdendo a função de guarda do lar.

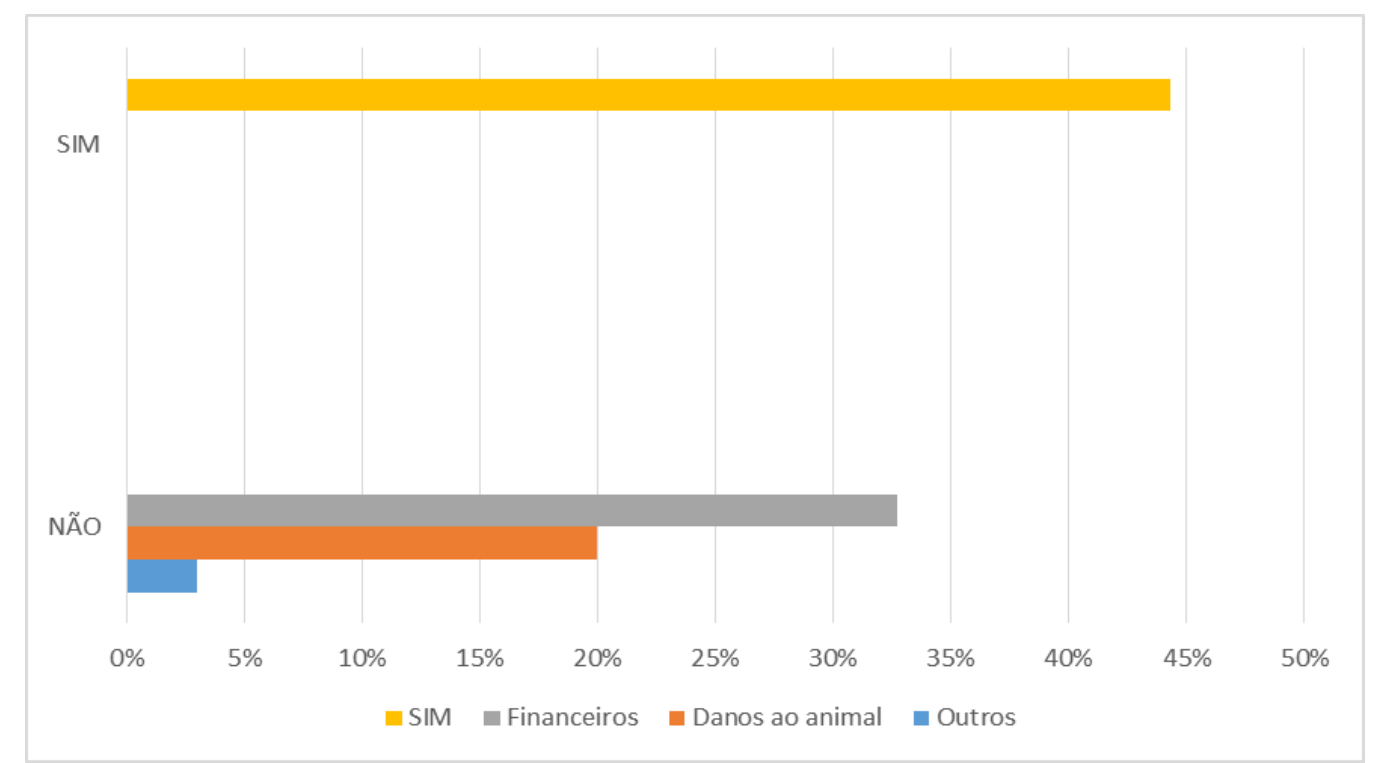

Gráfico 2. frequência sobre a opinião dos proprietários em realizar a cirurgia de castração em seus animais

\section{Conclusão}

Em relação a prática do uso de fármacos contraceptivos é de grande aceitação e de uso corriqueiro na cidade de Pedro II, e muitos desconhecem os efeitos adversos provocados pelo uso do fármaco em cães e gatos, principalmente pela carência de médicos veterinários atuando na cidade, que orientassem a respeito. Contudo notou-se que uma boa parcela realizaria castração se houvesse local para realização do procedimento cirúrgico na cidade, mostrando que o que necessita de antemão é de uma maior presença de médicos veterinários na região, além de clínica com suporte para realização de exames e cirurgias, assim como campanhas de castração para pessoas com baixa renda.

\section{Referências bibliográficas}

Bocardo, M., Dabus, D. M. M., Tentrin, T. C., Lima, G. S., \& Bariani, M. H. (2008). Influência hormonal na carcinogênese mamária em cadelas. Revista Científica Eletrônica de Medicina Veterinária, 6(11), 1-6.

Cáceres, L. P. N. (2004). Estudo do programa de esterilização das populações canina e felina no Município de São Paulo, período 2001 a 2003. Universidade de São Paulo. https://doi.org/10.11606/d.10.2004.tde-06072005-102729 
Dias, L. G. G. G., Oliveira, M. E., Dias, F. G. G., Calazans, S. G., \& Confort, V. A. (2013). Uso de fármacos contraceptivos e seus efeitos adversos em pequenos animais. Enciclopédia Biosfera, 9(16), 2077-2083.

Feldman, E. C., Nelson, R. W., Reusch, C., \& Scott-Moncrieff, J. C. (2014). Canine and feline endocrinology. Elsevier Health Sciences. https://doi.org/10.1016/c2010-0-67113-6

Fernandes, E. R. L., Costa, T. M., \& Leite, D. F. S. S. (2020). Uso de fármacos contraceptivos e seus efeitos colaterais em cães e gato: Revisão de literatura. Revista de Medicina VeterrináriaVII, 17(23), $1-14$.

Filgueira, K. D., Costa, P. F. C. R., \& Paula, V. V. (2008). Hiperplasia mamária felina: sucesso terapêutico com o uso do aglepristone. Ciência Animal Brasileira, 9(4), 1010-1016.

Fonseca, A. P. B., Moura, V. M., Chaves, R. A. A., Leite, E. R., \& Klein, R. P. (2014). Progestágenos para inibição do cio em cadelas e gatas vendidos em lojas veterinárias (p. 1067). ANAIS.

Gabaldi, S. H. (1998). Hiperplasia e prolapso vaginal em cadelas. Clínica Veterinária, 13, 17-18.

IBGE, Senso 2010. Cidade de Pedro II, Piauí. Disponível em: 〈http://cod.ibge.gov.br/237LY> Acessado em: 01 de Junho de 2019.

Johnston, S. D., Olson, M. V, Johnston, P. S. S. D., Kustritz, M. V. R., \& Olson, P. N. S. (2001). Canine and feline theriogenology. WB Saunders.

Kustritz, M. V. R. (2011). Clinical canine and feline reproduction: evidence-based answers. John Wiley $\&$ Sons.

Neves, M. M., Marques Júnior, A. P. \& Oliveira, E. C. S. (2003). Endocrinologia reprodutiva e controle da fertilidade da cadela - revisão. Archives of Veterinary Science, 8(1), 1-12.

Oliveira, E. C. S., \& Marques-Jr, A. P. (2006). Endocrinologia reprodutiva e controle da fertilidade da cadela. Revista Brasileira de Reprodução Animal, 30(1/2), 11-18. https://doi.org/10.5380/avs.v8i1.4007

Papich, M. G. (2012). Manual Saunders de Terapia Veterinária. Elsevier Health Sciences Brazil.

Romagnoli, S. (2003). Clinical approach to infertility in the queen. Journal of Feline Medicine and Surgery, 5(2), 143-146. https://doi.org/10.1016/s1098-612x(02)00131-6

Rossi, D., Guerrini, A., Paganetto, G., Bernacchia, G., Conforti, F., Statti, G., Maietti, S., Poppi, I., Tacchini, M., \& Sacchetti, G. (2013). Croton lechleri Müll. Arg. (Euphorbiaceae) stem bark essential oil as possible mutagen-protective food ingredient against heterocyclic amines from cooked food. Food Chemistry, 139(1-4), 439-447. https://doi.org/10.1016/j.foodchem.2013.01.076

Silva, F. A. N., Carvalho, R. L., Klein, R. P., \& Quessada, A. M. (2009). Posse responsável de cães no bairro Buenos Aires na cidade de Teresina (PI). ARS Veterinaria, 25(1), 14-17.

Viana, D. C., Santos, A. C., Rui, L. A., Oliveira, D. M., Silva, A. B., Costa, F. C. F. C., \& Assís Neto, A. C. (2012). Hiperplasia mamária felina: Um relato de caso. Veterinária Notícias, 18(2).

Recebido: 9 de março, 2020 Aprovado: 4 de abril, 2010

Disponível online: 6 outubro, 2020.
Licenciamento: Este artigo é publicado na modalidade Acesso Aberto sob a licença Creative Commons Atribuição 4.0 (CC-BY 4.0), a qual permite uso irrestrito, distribuição, reprodução em qualquer meio, desde que o autor e a fonte sejam devidamente creditados. 